\title{
Editorial
}

\section{Transfer und Transformationen von pädagogischem Wissen}

\section{Lucien Criblez und Rita Hofstetter}

Wissen war immer schon mobil, weil Menschen nicht sesshaft waren. Die Erfindung der Schrift hat das Wissen zunehmend verselbständigt, "weil sie es erlaubt zu kommunizieren, ohne da zu sein, zu erinnern, obwohl man vergessen hatte» (Kaube, 2017, S. 229). Die Erfindung der Schrift machte Wissen speicherbar, löste es von der gesprochenen Sprache, machte es potenziell ubiquitär nutzbar und konnte zumindest teilweise die Primärerfahrung ersetzen. Die «moderne» Schule, wie sie sich im 18. Jahrhundert abzuzeichnen begann und im 19. Jahrhundert in den westlichen Nationen als staatliche Aufgabe konsolidiert wurde, wäre ohne Schrift kaum möglich gewesen. Wir können sogar Kontinuitäten ins digitale Zeitalter feststellen, sind doch auch neue Technologien weitgehend schriftbasiert, auch wenn die Bedeutung von Bildern deutlich zugenommen hat.

Menschen und Geschriebenes, so liesse sich zugespitzt formulieren, waren während Jahrhunderten Träger des Wissens; durch die Digitalisierung wird diese Ausschliesslichkeit allerdings erweitert. Schulen waren die klassischen Orte, an denen Wissen gelehrt wurde (vgl. Burke, 2000, Kapitel 2) - daran wird sich wohl einiges ändern. Die Mobilität von Wissen ist heute nicht mehr einfach auf Menschen und Verschriftlichtes angewiesen. Das Wissen hat sich von ihren "klassischen» Trägern emanzipiert und kann über elektronische Träger schnell, global und potenziell allen gleichzeitig vermittelt werden. Das ist eine neue Dimension, die Schule und bisherige Bildungskonzepte zumindest herausfordern wird.

Die vorliegende Themennummer der Schweizerischen Zeitschrift für Bildungswissenschaften beleuchtet und problematisiert Fragen des Transfers und der Transformationen von schulischem bzw. pädagogischem Wissen, das über verschiedene Grenzen zirkuliert, unter sozialhistorischen Perspektiven und unter Berücksichtigung unterschiedlicher Ebenen. Dass Wissen mobil ist, wurde während Jahrhunderten insbesondere über mobiles Personal von Klöstern und Universitäten garantiert. Die eine Institution verstand sich global, weil der Glaube nicht an nationalstaatliche Grenzen gebunden ist, die andere, weil Wissenschaft sich schon 
sehr früh als globales «Unternehmen» verstand. Im Schulbereich kam es vor allem im Kontext der Staatsbildungsprozesse und insbesondere durch die Formierung von «Nationalstaaten» im 19. Jahrhundert zu einer Art Territorialisierung des Wissens: Das, was in Schulen gelehrt und gelernt werden soll, wurde zunehmend nationalstaatlich (in der Schweiz: kantonal) durch Schulgesetze, Lehrpläne und Lehrmittel normiert (Criblez, Giudici, Hofstetter, Manz \& Schneuwly, im Druck). Diese nationalstaatliche Orientierung von Bildungssystemen ist immer wieder durch Versuche, (national-)staatliche Grenzen zu überschreiten, relativiert worden (Droux \& Hofstetter, 2015; Fontaine, 2015; Matasci, 2015) - etwa durch Weltausstellungen im letzten Drittel des 19. Jahrhunderts (Dittrich, 2010; Gonon, 1999; Lawn, 2009), durch Bildungs- und Kinder-/Jugendschutzbestrebungen etwa im Rahmen des Völkerbundes (Droux, 2015; Fuchs, 2006a, 2007) und der UNO (Moody, 2016) oder durch international tätige Gremien wie die New Education Fellowship/Ligue Internationale pour l'Education nouvelle (Haenggeli-Jenni, 2017; Koslowski, 2013) oder das Bureau International d'Education (Hofstetter \& Schneuwly, 2013; Hofstetter, 2015).

Die nationalstaatliche Orientierung von Schule blieb auch nach dem Zweiten Weltkrieg erhalten, obwohl insbesondere Fragen der Bildungsplanung und der Öffnung der höheren Bildung in Gremien wie der United Nations Educational, Scientific and Cultural Organization (UNESCO) (1967) oder der Organisation for Economic Cooperation and Development (OCDE/OECD) (Bürgi, 2017; Jakobi \& Martens, 2010; OCDE, 1966; OECD 1967) spätestens seit den 1960er-Jahren in einem internationalen Kontext diskutiert wurden. Formen von "borrowing and lendig» (Steiner-Khamsi, 2004; Steiner-Khamsi \& Waldow, 2012) sind in diesem Sinne kein neues Phänomen, das sich im Zuge der internationalen PISA-Vergleichsstudien etablierte, sondern haben im Verlaufe der zweiten Hälfte des 20. Jahrhunderts stark zugenommen, insbesondere im Kontext der Bedeutungszunahme internationaler und transnationaler Organisationen (Herren, 2009).

Anders als von vielen zunächst erwartet war der West-Ost-Konflikt nach dem Mauerfall 1989 nicht wirklich zu einem Ende gekommen und auch «the end of history» (Fukuyama, 1989) war nicht erreicht. Zwar erleichterte das Ende des Kalten Krieges die sich bereits in den 1980er-Jahren deutlich abzeichnenden internationalen Liberalisierungs- und Globalisierungstendenzen, die zunächst ungebrochen, ab 9/11 und spätestens mit der Bankenkrise 2008 aber wieder relativiert wurden. Dass die "Ära» der Nationalstaaten nicht ihrem Ende entgegenging, wie unter Globalisierungs- und Internationalisierungsperspektiven immer wieder behauptet - oder vielleicht eher herbeigewünscht - worden war, hatte sich bereits in den Balkankriegen der 1990er-Jahre gezeigt und ist angesichts weltweiter nationalstaatlicher Überhöhungen inzwischen kaum mehr bestritten.

Sowohl in den Erziehungs- als auch in den Geschichtswissenschaften - und etwas verspätet deshalb auch in der Historischen Bildungsforschung - ist die stark auf nationalstaatliche Zusammenhänge ausgerichtete Forschung mit einer 
an internationalen Perspektiven orientierten Forschung ergänzt worden. Diese Perspektive wurde vor dem Hintergrund der Bolognareform und der international vergleichenden PISA-Studie weiter gestärkt, und auch die historische Bildungsforschung begann sich Ende der 1990er- und zu Beginn der 2000erJahre stärker mit Themen zu beschäftigen, die nicht einfach nur eine nationalstaatliche Perspektive nahelegten (Caruso \& Tenorth, 2002; Caruso, Koinzer, Mayer \& Priem, 2014; Droux \& Hofstetter, 2015; Fuchs, 2006b; Nordin \& Sundberg, 2014; Schriewer, 2016; Tröhler \& Lenz, 2015). Europäisierung (Lawn \& Grek, 2012), Internationalisierung (Caruso, 2014) und Globalisierung (Adick, 2002; Droux \& Hofstetter, 2015; Popkewitz \& Rizvi, 2009; Schriewer, 2007) wurden zu neuen und an Bedeutung gewinnenden Bezugspunkten der pädagogischen Forschung. Ob Schule und Bildung allerdings Teil einer «Weltkultur» werden (Meyer, 1997), in der sich die Bildungssysteme in Isomorphieprozessen allmählich immer stärker angleichen (Meyer \& Ramirez, 1997; für einen Überblick vgl. Hornberg, 2009) ist vielfach angezweifelt worden (u.a. Anderson-Levitt, 2003).

In diesem Kontext sind auch Konzepte anzusiedeln, mit denen die international vergleichende Perspektive der historischen Forschung neu profiliert wurde, insbesondere das Konzept der «histoire croisée» bzw. "Verflechtungsgeschichte» (Werner \& Zimmermann, 2002, 2006), mit dem die nationalstaatliche Engführung vergleichender Geschichtsschreibung überwunden werden sollte. Im Bereich der Erziehungswissenschaft wurde auch die kulturwissenschaftliche Variante des «transfert culturel» (Espagne, 1999) übernommen und für die Vergleiche und Transferprozesse auch in der historischen Bildungsforschung fruchtbar gemacht. Dieser Ansatz vertritt insbesondere das Postulat, dass mit jedem Transfer eine Metamorphose der Inhalte und Gegenstände, die zirkulieren, verbunden ist, weil die Wiederaneignung zu Bedeutungsverschiebungen führt. Espagne theoretisiert dies unter dem Begriff «resémantisation». Er zeigt übrigens auch, dass jede Kultur, auch wenn sie stark in einem spezifischen geographischen Raum und/oder Sprachraum verankert ist, immer schon das Produkt von Importen ist. Durch Zirkulationen unterschiedlicher Art entstehen Mischformen («métissages»). Durch die historische Rekonstruktion lassen sich solche Prozesse der zunehmenden Hybridisierung entschlüsseln (Espagne, 2013). Dieser Ansatz ist von verschiedenen Historikerinnen und Historikern eingesetzt worden um aufzuzeigen, wie pädagogische Konzepte, Lehrmittel, pädagogische Ideen usw. von einem Kulturraum in einen andern transferiert werden (vgl. insbesondere Darme, 2018; de Mestral, 2018; Fontaine, 2015; Gevaert, 2017; Gispert \& Schubring, 2011; Rouiller, 2018).

Vor diesem Hintergrund beschäftigt sich die vorliegende Themennummer mit den Transfers und Transformationen von pädagogischem Wissen in unterschiedlichen Kontexten und auf unterschiedlichen Analyseebenen, wobei vor allem kulturelle Transfers im Bildungsbereich thematisiert werden. Transfers werden zwischen unterschiedlichen Nationalstaaten, zwischen supranatio- 
nalen Organisationen und Nationalstaaten, zwischen Nationalstaaten und Nachbar-Teilstaaten, zwischen den Teilstaaten (Kantonen) sowie zwischen Bundesstaat und Nationalstaat beobachtet.

Martin Lawn berichtet über die erstaunliche internationale Ausbreitung einer pädagogischen Innovation im letzten Drittel des 19. Jahrhunderts, die im begrenzten Raum eines kleinen schwedischen Dorfes (Nääs) entstanden war: die Einführung des Handarbeitsunterrichts in der Schule. Er stützt sich dabei auf das Konzept des transnational turn (speziell in der Lesart von Akira Iriye und Pierre-Yves Saunier) und überprüft die «Schalthebel» und Förderungsstrategien, die der Sloyd-«Erfinder», Otto Salomon, und seine Schüler als Netzwerker einsetzten, um eine eigentliche Denkschule rund um das Schulfach zu etablieren. Weltausstellungen, Lehrerverbände und wissenschaftliche Gesellschaften dienten ebenso als bevorzugte Transferagenturen wie Schulbücher sowie Publikationen und deren Übersetzungen, um am Übergang vom 19. zum 20. Jahrhundert (manchmal auch als erste Globalisierungsära bezeichnet) Erfahrungen aus dem pädagogischen Experiment bekannt zu machen und im grossen Stil zu verbreiten. Nääs funktionierte für die pädagogische Innovation auch als eigentliches Epizentrum: Es zog Studierende aus der ganzen Welt an, die sich ausbilden liessen und dadurch zu Transporteuren von Sloyd über Grenzen und Kontinente hinweg wurden. Eine Handwerker- und Mittelschule, die Sloyd Training School, entstand am Ende des 19. Jahrhunderts sogar in Boston und ermöglichte die Ausbildung von Hunderten von Lehrern, welche die Methode anschliessend in ganz Amerika verbreiteten.

Das Interesse an einem solchen Ansatz liegt auch darin, einerseits Phänomene der Aneignung und der Bedeutungsverschiebung und andererseits Prozesse der An- und Einpassung in neuen Kontexten im Zuge eines Transfers von Innovationen zu analysieren - im konkreten Fall ausgehend von Nääs und zirkulierend in andern europäischen Ländern, sogar in andern Kontinenten: Asien, Afrika und den beiden Amerika. Das Beispiel zeigt auch, dass die grossen Staaten in dieser Epoche ihre Bildungssysteme, ihre pädagogischen Praktiken und ihre pädagogischen Prinzipien stark unter Bezugnahme auf ausländische Erfahrungen entwickelt haben. Das berücksichtigte Schulfach Handarbeit ist zusätzlich von Bedeutung, weil sich dafür nicht nur Schulprotagonisten (Familien, Lehrpersonen, Bildungsadministrationen) einsetzten, sondern auch Industrielle und Exponenten der ökonomischen Welt, die darauf insistierten, dass das sogenannte Handarbeiten an den Bedürfnissen des Marktes ausgerichtet werde, Bedürfnissen notabene, die dem Konjunkturwandel unterworfen sind.

Wie bedient sich eine sprachliche Minderheit in einem Land der pädagogischen Ideen und Konzepte des grossen Nachbarlandes, mit dem es eine sprachkulturelle Einheit bildet? Wolfgang Sahlfeld zeigt dies am Beispiel von pädagogischen Transfers aus Italien in den Kanton Tessin im ersten Drittel des 20. Jahrhunderts. In seinem Beitrag untersucht er, wie reformpädagogische Ideen, u.a. von Maria Montessori und Giuseppe Lombardo-Radice, in der 
Bildungspolitik und im öffentlichen Diskurs über Schule und Unterricht, ja in den Lehrplänen im Kanton Tessin aufgenommen wurden. Italien galt im Tessin zunächst als Kulturnation, der sich der Kanton Tessin zugehörig fühlte, die gerade in ihren reformpädagogischen Diskursen als fortschrittlich galt und deren pädagogische Konzepte es nachzuahmen galt. Dass italienische Experten im Tessin eine Art Beraterfunktion übernahmen, ist wohl einer der wirksamsten Transfermechanismen gewesen. Reformpädagogisches Handeln im Kanton sei deshalb nur durch den italienischen Einfluss zu erklären. Sahlfeld stellt aber nicht nur die Frage nach den Funktionsweisen von kulturellem Transfer, sondern fragt auch, ob und wie diese Transfers öffentlich thematisiert worden sind.

Nach Sahlfeld sind im Hinblick auf pädagogische Transfers zwei Phasen zu unterscheiden. Für die erste Phase (bis etwa 1915) zeigt er auf, dass beim «metodo intuitivo", einer Methode, die im Tessin als schweizerische "Erfindung» charakterisiert wurde, als die einzig wissenschaftlich legitimierte Methode galt und deshalb in allen Schulfächern zur Anwendung kommen sollte, sowohl die italienische Herkunft als auch die Anlehnungen an die Herbart-Zillersche Methode verschwiegen wurden. Die zweite Phase sei dann ganz klar geprägt gewesen von reformpädagogischen Konzepten, insbesondere italienischer Herkunft (Montessori), die im Tessin u.a. durch Teresa Bontempi und Maria Boschetti-Alberti rezipiert und den lokalen Verhältnissen angepasst wurden. Letztlich weist Sahlfeld auf den grundlegenden Konflikt in der Tessiner Schulpolitik und Pädagogik hin, der dadurch entstanden war, dass die Schule im Kanton Tessin zwar der durch die katholische Religion und die italienische Sprache geprägten italienischen Kulturtradition verpflichtet war, sich gleichzeitig aber, als Teil der demokratisch-liberalen Schweiz, auch gegen den italienischen Faschismus abgrenzen musste. Daraus liesse sich schliessen: Nicht nur die kulturelle Tradition und Zugehörigkeit, sondern auch das politische Selbstverständnis sind für die Konzeption von Schule von Bedeutung.

Stark abgestützt auf das Konzept und die Methodologie des transfert culturels untersucht der Beitrag von Alexandre Fontaine, Aurélie de Mestral und Viviane Rouiller die interkantonale Zirkulation von schulischem Wissen an ausgewählten Schulbüchern, die in der Schweiz in den letzten 200 Jahren verwendet wurden. Der Autor und die Autorinnen richten ihre Aufmerksamkeit auf drei Schulfächer, die in unterschiedlicher Art und Weise zur Konsolidierung kantonaler, regionaler und nationaler Identität beitragen sollen: Geschichte, Politische Bildung und Deutsch. Sie untersuchen in verschiedenen Westschweizer Kantonen empfohlene und in den Schulen eingeführte Schulbücher auf die Bedeutungsverschiebungen bei der Einführung hin - was sie als «Übersetzungen» («translations») bezeichnen. Sie zeigen auf, dass sich bei den Geschichtsbüchern Transformationsprozesse beobachten lassen, wenn zwei oder drei Kantone dieselben Schulbücher verwenden, weil die Inhalte und die Verwendungsweisen (wie sie etwa in den Vorwörtern beschrieben sind) je nach Kontexten angepasst werden. Untersucht wurden 20 Schulbücher aus einer 
Sammlung von 164, die für die Kantone Freiburg, Genf und Waadt inventarisiert worden waren. Für die Untersuchung der Politischen Bildung lag der Fokus auf der Analyse der "Übersetzungen», die in den verschiedenen Schulbüchern beobachtet werden konnten, wenn sie von einer reformierten in eine katholische Region oder von einer konfessionell orientierten in eine säkularisierte bzw. republikanische Gemeinschaft transferiert wurden. Ein typisches Beispiel dafür sind die Schulbücher von Louis Bornet, eines der ersten zur Politischen Bildung in der Dritten Republik, und von Numa Droz. Auch wenn die Mehrheit der Schulbücher für Deutsch als Fremdsprache in denselben Kantonen produziert wurden, in denen sie auch verwendet wurden, sind einige unter ihnen doch Resultat von Transfers, an denen sich «Übersetzungen» beobachten lassen, so zum Beispiel der Deutschkurs von Léopold Rochat und Anne Lohmann, der im Beitrag untersucht wird. Die Analyse der Verflechtungen zwischen Lehrmitteln aus der Schweiz und aus Deutschland zeigt sowohl methodische Anleihen (zum Teil auch versteckte Übernahmen und Mischformen) als auch eine «Helvetisierung» der Inhalte, von Geschichten, die den Westschweizer Schülerinnen und Schülern zur Lektüre vorgelegt werden.

Die kulturellen Transfers, dies zeigen die Untersuchungen von Alexandre Fontaine, Aurélie de Mestral und Viviane Rouiller, wurden in den drei Schulfächern unterschiedlich realisiert. Die Analyse weist auf die Dichte der Transfers, aber auch auf die Labilität und Instabilität von Austausch und Anleihen über die konfessionellen, kulturellen, sprachlichen und nationalen Grenzen hinweg hin. Insgesamt stellt die Untersuchung aber den heuristischen Wert einer Verflechtungsgeschichte unter Beweis, die darauf abzielt, sich von dem, was die Autoren die Obsession eines differenzialistischen Erbes («obsession différencialiste héritée») des 19. Jahrhunderts nennen, loszulösen zugunsten eines Ansatzes, der die Verflechtungen des Lokalen und Prozesse der Vermischung und Transformationen im Transferprozess in den Blick nimmt.

Die Curriculumdebatte der späten 1960er- und der 1970er-Jahre um die Neukonzeption von Lehrplänen war eine international geführte Debatte, die aber stark von Entwicklungen in der amerikanischen Psychologie beeinflusst war. Eines der deutschsprachigen Zentren dieser Debatte entstand an der Universität Freiburg in der Schweiz um Karl Frey, der später seine Arbeiten als Direktor des Instituts für Pädagogik der Naturwissenschaften in Kiel fortsetzte. Lukas Höhener und Lucien Criblez zeigen in ihrem Beitrag auf, wie die Freiburger Arbeitsgruppe für Lehrplanforschung (FAL) durch unterschiedliche Transfers ihre Bedeutung erlangte und sich zu einer der ersten «modernen» erziehungswissenschaftlichen Forschergruppen im deutschen Sprachraum entwickeln konnte - die allerdings nicht allzu lange Bestand hatte.

In einem ersten Schritt zeigt der Beitrag auf, wie die FAL sich in ihrem Konstituierungsprozess in der zweiten Hälfte der 1960er-Jahre vor allem amerikanischer psychologischer Theorien bediente, um Fragen der Curriculumtheorie und -entwicklung neu zu fundieren und wissenschaftlich zu legitimieren. Der 
Erfolg lag darin, dass die Theorien, Konzepte und Modelle nicht einfach nur transferiert, sondern auch transformiert und auf die neuen Kontexte angepasst wurden. Dies war nicht zuletzt möglich, weil die Lehrplanentwicklung stark auf die Mitwirkung der pädagogischen Praxis hin angelegt war. Gleichzeitig konnte die Lehrplanarbeit stärker am breiteren Selbstverständnis der angelsächsischen curriculum studies orientiert werden. Ein zweiter Transfer fand statt, indem die meisten Vertreter der FAL in den 1970er-Jahren von der Wissenschaft in die Bildungsadministration oder in die Lehrerinnen- und Lehrerbildung wechselten, wo sie weiterhin ihre Ideen und Projekte im Curriculumbereich verfolgten. Einen dritten Transfermechanismus sehen die Autoren in der Beratertätigkeit: Die Mitglieder der FAL waren in den 1970er-Jahren in sehr unterschiedlichen Reformbereichen beratend tätig. Der Beitrag schliesst mit kritischen Fragen, unter anderem: inwiefern mit den beiden letzten Transfers die Grenzen zwischen Wissenschaft und Politik aufgelöst - oder zumindest relativiert - wurden.

Einen gescheiterten Versuch eines «forcierten» Kulturtransfers fokussieren Anja Giudici und Karin Manz in ihrem Beitrag zur nationalen Erziehung zwischen 1914 und 1924. Einen wesentlichen politischen Ausgangspunkt bildet die Situation der Schweiz im Ersten Weltkrieg, in der die französischsprachige Schweiz mit Frankreich und die deutschsprachige Schweiz mit Deutschland sympathisierten. Bereits kurz nach Kriegsausbruch kam es deshalb zu politischen Konflikten zwischen den beiden grossen Sprachregionen in der Schweiz. Die drohende Spaltung der Schweiz sollte mit einem nationalpädagogischen Programm, das Schweizergeschichte, Politische Bildung und die schweizerischen Landessprachen umfassen sollte, abgewendet werden. Im föderalistischen Bildungssystem sollten deshalb nationale Vorgaben Einzug finden. Der politisch dominierende Freisinn versuchte also, ein solches Programm für die ganze Schweiz verpflichtend zu machen. Sowohl der katholisch-konservative Teil der Schweiz als auch die Sozialdemokratie, aber auch Lehrerverbände und Kantone opponierten gegen das Programm, das dann tatsächlich aus der politischen Agenda entfernt wurde. Während die einen im sogenannten Programm "Wettstein-Calonder» einen Angriff auf ihre kulturelle, vor allem katholisch geprägte Identität sahen, erachteten die andern das Programm als zu nationalistisch und forderten eine stärker internationale Orientierung der schulischen Programme. Letztlich handelte es sich bei der nationalen Erziehung um ein Programm, das zentralstaatlich verordnet werden sollte und dadurch den Bildungsföderalismus, der seit dem 19. Jahrhundert in der Schweiz immer schon kulturpolitisch (Sprache und Religion) legitimiert worden war, in Frage stellte. Der Beitrag ist denn auch ein gutes Beispiel dafür, wie «forcierter» Kulturtransfer verhindert wird. Er zeigt aber auch auf, dass Transfers auch von der gegenseitigen Bereitschaft abhängig sein können, Kultur überhaupt vom einen zum andern Ort zu transferieren.

Der Beitrag der Wissenschaftshistorikerin Hélène Gispert beschäftigt sich mit Mathematikzeitschriften, die sich an Lehrerinnen und Lehrer als Lesepublikum 
wenden und in sehr unterschiedlichen kulturellen Kontexten in Europa seit der Mitte des 18. Jahrhunderts herausgegeben wurden. Die Autorin fordert auf, die Mathematik so, wie sie in unterschiedlichen Wissenschafts- und Schulbereichen praktiziert wurde, zu entdecken, indem der Unterricht als spezifische Form der Zirkulation von Wissen betrachtet wird. Die minutiöse Analyse der editorischen Auswahl und der Inhalte der Zeitschriften zeigt, dass das gemischte berufliche Publikum - namentlich Studierende, Lehrerinnen und Lehrer, Professorinnen und Professoren, Hauslehrer, professionelle und Laiennutzer - ebenfalls an der Fabrikation und Transmission von mathematischem Wissen beteiligt ist. Dies zeigt sich vor allem in der während langer Zeit abgedruckten Rubrik «Fragen Antworten", die konzipiert wurde, um Vertrauen beim Publikum zu gewinnen, es zu erweitern und so die Zeitschrift abzusichern, aber auch, um die Beiträge auf die Schülerschaft abzustimmen, wie Gispert präzisiert. Indem sie aufzeigt, wie Lesegemeinschaften sich engagierten, um Mathematik in den Spalten der erwähnten Mathematikzeitschriften zu diskutieren, kann die Autorin nachweisen, dass insbesondere die Lehrer Mathematik eigentlich kokonstruieren, indem durch ihre Mitautorschaft mathematisches Wissen produziert und über die Zeitschriften verbreitet wird. Dadurch kann die Autorin für den untersuchten Zeitraum die traditionelle Unterscheidung zwischen wissenschaftlichen und professionellen Sphären bzw. die Dichotomie zwischen wissenschaftlichem und schulischem Wissen in Frage stellen. Indem sie die editorischen Leistungen der Jahre 1830-1840 untersucht, für eine Zeit also, in der die mathematische Öffentlichkeit eine Differenzierung erfährt, die mit derjenigen im Schulsystem verbunden ist, kann sie zeigen, dass eine sogenannte intermediäre Mathematik entsteht: relevant für die Forschung, aber elementarisiert, um besser zugänglich zu sein. Es handelt sich dabei offensichtlich um eine editorische Strategie, die sich um die Jahrhundertwende vom 19. zum 20. Jahrhundert in Europa ausbreitete. Diese Entwicklung steht gleichzeitig für eine Form der Internationalisierung editorischer Konzepte - auch wenn sich die Mathematikzeitschriften für Lehrer an Praktiker wenden, die in einem lokalen und klar begrenzten Rahmen unterrichten. Die 1899 durch den Genfer Henri Fehr und den Pariser Charles-Ange Laisant initiierte Zeitschrift «L'enseignement mathématique» etwa unterschied sich von andern durch ihren universalistischen und progressiven Geist und ist ein gutes Beispiel dafür, wie im beginnenden 20. Jahrhundert die Debatten und Untersuchungen zum Mathematikunterricht auf eine wirklich globale Ebene angehoben wurden.

Der Fremdsprachenunterricht wurde in der Schweiz in den 1970er-Jahren massgeblich reformiert: Nicht nur sollten Fremdsprachen früher unterrichtet werden, sondern auf der Sekundarstufe I sollten in Zukunft alle Schülerinnen und Schüler in mindestens einer Fremdsprache, die gleichzeitig aber eine Schweizer Landessprache sein sollte, unterrichtet werden. Sandra Grizelj und Daniel Wrana zeigen in ihrem Beitrag auf, dass diese Eckwerte der schulischen Fremdsprachenpolitik nicht einfach in der Schweiz erfunden wurden, sondern 
einer Forderung des Europarates entsprachen, der die Verständigung in Europa über die Verbesserung der Sprachkompetenzen fördern wollte. Zunächst rekonstruieren die Autorin und der Autor deshalb die Sprachenpolitik des Europarates, dem die Schweiz 1963 beigetreten war. Dann führen sie die neue Fremdsprachenpolitik am Beispiel der Empfehlungen der Schweizerischen Konferenz der kantonalen Erziehungsdirektoren (EDK) zum Fremdsprachenunterricht 1975 aus. Da sich diese Empfehlungen explizit an den «Vorgaben» des Europarates orientierten, gehen die Autorin und der Autor davon aus, dass ein kultureller Transfer von einer supranationalen Instanz auf die nationale Ebene stattgefunden hat. Zugleich können sie zeigen, dass sich die Kantone erstmals ernsthaft als aussenpolitischer Akteur zu positionieren versuchten, weil sie für den Sprachunterricht in der Schule zuständig sind.

Der Beitrag zeigt aber auch, dass Transfers auf Transferinstanzen angewiesen sind; in diesem Fall war es innerhalb der Schweiz die EDK: Die Empfehlungen richteten sich an die Kantone, die sie in ihren Lehrplänen und Stundentafeln umsetzen sollten. Die Koordination sollten die vier Regionalkonferenzen der EDK übernehmen. In diesem Transferprozess kam es jedoch zu einer Transformation der Zielsetzungen: Das ursprünglich formulierte Ziel der Verbesserung der Kommunikationsfähigkeit innerhalb der Schweiz bzw. innerhalb Europas wurde zunehmend von Problemen der Umsetzung in den Kantonen überlagert. Auch wenn die hochgesteckten Ziele wohl nicht erreicht werden konnten, kann der damalige Transfer von Ideen eines verbesserten Fremdsprachenlernen als Beginn einer Entwicklung angesehen werden, die letztlich in den Gemeinsamen Europäischen Referenzrahmen für Sprachen (GER) mündete. Dass wesentliche Grundlagen für diesen GER in der Schweiz entwickelt wurden, weist darauf hin, dass Transferprozesse oft nicht nur in eine Richtung zeigen.

\section{Bibliographie}

Adick, C. (2002). The impact of globalisation on national education systems. In C. Wulf \& C. Merkel (Hrsg.), Globalisierung als Herausforderung der Erziehung. Theorien, Grundlagen, Fallstudien (S. 45-58). Münster: Waxmann.

Anderson-Levitt, K. M. (2003). A world culture of schooling? In K. M. Anderson-Levitt (Ed.), Local meanings, global schooling (S. 1-26). New York: Palgrave Mcmillan.

Bürgi, R. (2017). Die OECD und die Bildungsplanung der freien Welt. Denkstile und Netzwerke einer internationalen Bildungsexpertise. Opladen: Budrich.

Burke, P. (2000). A social history of knowledge. Cambridge: Blackwell.

Caruso, M. (2014). Within, between, above, and beyond: (Pre)positions for a history of the internationalisation of educational practices and knowledge. Paedagogica historica, 50, (1-2), 10-26.

Caruso, M., Koinzer, T., Mayer, C. \& Priem, K. (Hrsg.) (2014). Zirkulation und Transformation. Pädagogische Grenzüberschreitungen in historischer Perspektive. Köln: Böhlau.

Caruso, M. \& Tenorth, H.-E. (Hrsg.) (2002). Internationalisierung - Internationalisation. Semantik und Bildungssystem in vergleichender Perspektive. Frankfurt/M.: Lang.

Criblez, L., Giudici, A., Hofstetter, R., Manz, K. \& Schneuwly, B. (Hrsg.) (im Druck). Die schulische Wissensordnung im Wandel. Schulfächer, Lehrpläne, Lehrmittel. Zürich: Chronos. 
Darme, A. (2018). Enseigner la grammaire pour développer l'expression de la pensée? Éléments d'histoire de la grammaire scolaire en Suisse romande (1830-1990). Thèse de Doctorat en Sciences de l'éducation, Université de Genève.

De Mestral, A. (2018). Enseigner l'histoire en Suisse romande et édifier la nation helvétique? Evolution d'un savoir scolaire à l'aune des programmes et des manuels (XIXe-XXe siècles). Thèse de Doctorat en Sciences de l'éducation, Université de Genève.

Dittrich, K. (2010). Experts going transnational: Education at world exhibitions during the second half of the nineteenth century. Thesis of Philosophy, University of Portsmouth.

Droux, J. (2015). Children and youth: A central cause in the circulatory mechanisms of the League of Nations (1919-1939). Prospects, 45, (1), 63-76.

Droux, J. \& Hofstetter, R. (Éd.) (2015). Globalisation des mondes de l'éducation. Circulations, connexions, réfractions, XIXe-XXe siècles. Rennes: Presses universitaires.

Espagne, M. (1999). Les transferts culturels franco-allemands. Paris: PUF.

Espagne, M. (2013). La notion de transfert culturel. Revue Sciences/Lettres, 1. Consulté le 9 avril 2018 dans http://rsl.revues.org/219; DOI: 10.4000/rsl.219

Fontaine, A. (2015). Aux heures suisses de l'école républicaine. Un siècle de transferts culturels et de déclinaisons pédagogiques dans l'espace franco-romand. Paris: Demopolis.

Fuchs, E. (2006a). Kinderschutz und Völkerbund: Zur Formierung des edukativen Multilateralismus in der Zwischenkriegszeit. In E. Fuchs (Hrsg.), Bildung international. Historische Perspektiven und aktuelle Entwicklungen (S. 163-180). Würzburg: Ergon.

Fuchs, E. (Hrsg.) (2006b). Bildung international. Historische Perspektiven und aktuelle Entwicklungen. Würzburg: Ergon.

Fuchs, E. (2007). The Creation of New International Networks in Education: The League of Nations and Educational Organizations in den 1920s. Paedagogica Historica, 43, (2), 199-209.

Fukuyama, F. (1989). The End of History? The National Interest, Summer 1989, 1-18. Zugriff am 03.04.2018 unter https://www.embl.de/aboutus/science_society/discussion/ discussion_2006/ref1-22june06.pdf

Gevaert, H. (2016). La Loi Guizot du 28 juin 1833, une sortie de l'ancien régime scolaire? Recherche sur l'organisation pédagogique des classes et les écoles primaires supérieures jusqu'à l'enseignement secondaire spécial de Victor Duruy. Thèse de Doctorat en Sciences de l'éducation, Université de Caen.

Gispert, H. \& Schubring, G. (2011). Societal, structural and conceptual changes in mathematics teaching: Reform processes in France and Germany over the twentieth century and the international dynamics. Science in context 24, (1), 73-106.

Gonon, Ph. (1999). Weltausstellungen im 19. Jahrhundert als Ansporn für Schulreformen. In L. Criblez, R. Hofstetter, C. Jenzer \& Ch. Magnin (Hrsg.), Eine Schule für die Demokratie (S. 377-401). Bern: Lang.

Haenggeli-Jenni, B. (2017). L'Éducation nouvelle entre science et militance. Débats et combats à travers la revue "Pour l'Ére Nouvelle» (1920-1940). Berne: Lang.

Herren, M. (2009). Internationale Organisationen seit 1865. Eine Globalgeschichte der internationalen Ordnung. Darmstadt: Wissenschaftliche Buchgesellschaft.

Hofstetter, R. (2015). Building an «international code for public education»: Behind the scenes at the International Bureau of Education (1925-1946). Prospects, XLV, 31-48.

Hofstetter, R. \& Schneuwly, B. (2013). The International Bureau of Education (1925-1968): A platform for designing a «chart of world aspirations for education». European Educational Research Journal, 12, (2), 215-230.

Hornberg, S. (2009). Potential of the world polity approach and the concept «Transnational Spaces» for the analysis of new developments in education. Journal for Educational Research Online, 1, (1), 241-253. 
Jakobi, A. P. \& Martens, K. (2010). Expanding and intensifying governance: The OECD in education policy. In K. Martens \& A. P. Jakobi (Eds.), Mechanism of OECD Governance (S. 163-179). Oxford: Oxford University Press.

Kaube, J. (2017). Die Anfänge von allem. Berlin: Rowohlt.

Koslowski, S. (2013). Die New Era der New Education Fellowship. Ihr Beitrag zur Internationalität der Reformpädagogik im 20. Jahrhundert. Bad Heilbrunn: Klinkhardt.

Lawn, M. (Ed.). (2009). Modelling the future. Exhibitions and the materiality of education. Oxford: Symposium Books.

Lawn, M. \& Grek, S. (2012). Europeanizing education. Oxford: Symposium Books.

Matasci, D. (2015). L'école républicaine et l'étranger. Paris: ENS Edition.

Meyer, J. W. \& Ramirez, F. O. (1997). Die globale Institutionalisierung von Bildung. In J. W. Meyer, Weltkultur (S. 212-234). Frankfurt/M.: Suhrkamp.

Meyer, J. W. (1997). Weltkultur. Frankfurt/M.: Suhrkamp.

Moody, Z. (2016). Les Droits de l'enfant. Genèse, institutionalisation et diffusion (1924-1989). Neuchâtel: Edition Alphil.

Nordin, A. \& Sundberg, D. (Eds.) (2014). Transnational policy flow in european education. The making and governing of knowledge in the education policy field. Oxford: Symposium Books.

OCDE (1966). Planification de l'enseignement. Problèmes d'organisation. Paris: OCDE.

OECD (1967). Begabung und Bildungschancen. Frankfurt/M.: Diesterweg. (engl. Original: 1961)

Popkewitz, Th. S. \& Rizvi, F. (Eds.) (2009). Globalization and the study of education. YearBook of the National Society for the Study of Education. Oxford: Blackwell Publishers.

Rouiller, V. (2018). Apprendre la langue de la majorité des Confédérés. Une discipline scolaire, entre enjeux pédagogiques, politiques, pratiques et culturels (1830-1990). Thèse de Doctorat en Sciences de l'éducation, Université de Genève.

Schriewer J. (Hrsg.) (2007). Weltkultur und kulturelle Bedeutungswelten. Zur Globalisierung von Bildungsdiskursen. Frankfurt/M.: Campus.

Schriewer, J. (Ed.) (2016). World culture re-contextualised: Meaning constellations and path-dependencies in comparative and international education research. London: Routledge.

Steiner-Khamsi, G. (2004). The global politics of educational borrowing and lending. New York, NY: Teachers College Press.

Steiner-Khamsi, G. \& Waldow, F. (Éd.) (2012). Policy brrowing and lending in education. World Yearbook of Education 2012. London: Routledge.

Tröhler, D. \& Lenz, T. (Eds.) (2015). Trajectories in the development of modern school systems. Between the national and the global. New York: Routledge.

UNESCO. (1967). Perspektiven der Bildungsplanung. Frankfurt: Diesterweg.

Werner, M. \& Zimmermann, B. (2002). Vergleich, Transfer, Verflechtung. Der Ansatz der Histoire croisée und die Herausforderung des Transnationalen. Geschichte und Gesellschaft, 28, 607-636.

Werner, M. \& Zimmermann, B. (2006). Beyond comparison. Histoire croisée and the challenge of reflexivity. History and Theory, 45, 30-50. 
